\title{
Télescope
}

Revue d'analyse comparée en administration publique

\section{Positionnement institutionnel et innovation dans le secteur public}

\section{Luc Bernier, Taïeb Hafsi et Carl Deschamps}

Volume 19, numéro 2, printemps 2013

L'innovation dans le secteur public : au-delà des discours

URI : https://id.erudit.org/iderudit/1023839ar

DOI : https://doi.org/10.7202/1023839ar

Aller au sommaire du numéro

Éditeur(s)

L'Observatoire de l'administration publique

ISSN

1929-3348 (numérique)

Découvrir la revue

Citer cet article

Bernier, L., Hafsi, T. \& Deschamps, C. (2013). Positionnement institutionnel et innovation dans le secteur public. Télescope, 19(2), 38-56.

https://doi.org/10.7202/1023839ar

\section{Résumé de l'article}

Dans le système de gouvernement britannique, il existe trois types d'organisations : les organismes centraux qui sont responsables de la coordination de l'appareil gouvernemental, les ministères qui gèrent les domaines liés aux politiques et les organisations autonomes qui sont dotées de missions plus spécialisées. On peut émettre une hypothèse selon laquelle les organismes centraux, étant à proximité des politiques, risquent d'être plus conservateurs en raison de leur tendance naturelle à éviter les risques et les questions embarrassantes pour les ministres desquels ils relèvent. Serait-il possible que l'éloignement des politiques favorise un plus grand espace pour innover ? Le présent article teste cette hypothèse à l'aide de données obtenues dans les candidatures au Prix de la gestion innovatrice de l'Institut d'administration publique du Canada. Excepté le cas des entreprises publiques, dont les résultats n'étaient pas significatifs, l’hypothèse est confirmée. La création d'agences gouvernementales permet l'innovation. 


\title{
POSITIONNEMENT INSTITUTIONNEL ET INNOVATION DANS LE SECTEUR PUBLIC
}

\author{
Par Luc Bernier, Professeur, École nationale d'administration publique \\ luc.bernier@enap.ca \\ Taïeb Hafsi, Professeur, HEC-Montréal \\ taieb.2.hafsi@hec.ca \\ Et Carl Deschamps, Étudiant, ENAP Québec \\ carl.deschamps@enap.ca \\ Traduit de l'anglais
}

RÉSUMÉ Dans le système de gouvernement britannique, il existe trois types d'organisations : les organismes centraux qui sont responsables de la coordination de l'appareil gouvernemental, les ministères qui gèrent les domaines liés aux politiques et les organisations autonomes qui sont dotées de missions plus spécialisées. On peut émettre une hypothèse selon laquelle les organismes centraux, étant à proximité des politiques, risquent d'être plus conservateurs en raison de leur tendance naturelle à éviter les risques et les questions embarrassantes pour les ministres desquels ils relèvent. Serait-il possible que l'éloignement des politiques favorise un plus grand espace pour innover? Le présent article teste cette hypothèse à l'aide de données obtenues dans les candidatures au Prix de la gestion innovatrice de l'Institut d'administration publique du Canada. Excepté le cas des entreprises publiques, dont les résultats n'étaient pas significatifs, l'hypothèse est confirmée. La création d'agences gouvernementales permet l'innovation.

ABSTRACT In the Westminster tradition of government, there are three types of organizations: the central agencies that coordinate the entire state apparatus, the departments that manage policy domains and the more autonomous agencies that have more specialized missions. The hypothesis could be made that the central agencies are closer to politics and more likely to be more conservative, trying to avoid risk and questions to the ministers. Is it possible that being further from politics allows having more space to innovate? Using data from the Canadian Management Innovation Award, we test this hypothesis. Apart of the results on public enterprises where the results are not significative, the hypothesis is confirmed. Agentification allows innovation. 
Dans le système de gouvernement britannique, on compte trois principaux types d'organisations : les organismes centraux qui sont chargés de la coordination de l'appareil gouvernemental dans son ensemble, les ministères qui assument des responsabilités fonctionnelles dans des domaines tels que l'éducation, la santé, l'urbanisme, et divers organismes autonomes comme les agences, les entreprises publiques, les organismes de réglementation, etc. Ces derniers sont organisés selon leur fonction de base et ils sont suffisamment éloignés du contrôle politique pour mener à bien leurs activités quotidiennes sans trop de supervision des organismes centraux. Certains gouvernements ont laissé à ces organisations une plus grande autonomie, car elles n'élaborent pas de politiques; elles sont habituellement responsables de la prestation de services particuliers. Dans ce système politique présent au Royaume-Uni, mais également dans les anciens dominions que sont l'Australie, le Canada et la Nouvelle-Zélande, depuis les réformes du XIXe siècle, les ministres sont tenus responsables devant le Parlement des succès et des échecs des fonctionnaires qu'ils sont censés superviser. Étant donné la taille et la complexité des gouvernements modernes, cette responsabilité est devenue plus un principe qu'une réalité. Or le système engendre une sorte de pression centralisatrice afin d'éviter les erreurs qui pourraient embarrasser le ministre responsable d'un ministère. On pourrait prétendre que plus on se trouve près du cœur du gouvernement, plus on tentera d'éviter les risques et plus on tâchera de respecter les règles. Les possibilités d'innovation s'en trouvent restreintes.

Dans le cadre des réformes des trois dernières décennies, les gestionnaires étaient censés obtenir plus d'autonomie. La gestion par résultats devait à priori remplacer les contrôles de l'administration publique traditionnelle. On pourrait croire que les ministères centraux de nombreux gouvernements ont eu tendance à conserver les anciens mécanismes de contrôle tout en ajoutant les nouveaux, ce qui a rendu encore plus difficiles les tentatives de gestion hors des règles établies. De plus, la crise économique qui dure depuis 2008 rend la situation encore plus pénible et nombre de pays risquent de continuer à y faire face dans l'avenir immédiat. Au départ, cette crise a forcé les gouvernements à renflouer le système bancaire et à dépenser d'importantes sommes pour conserver la vitalité de leurs systèmes économiques. Les montants dépensés ont ensuite poussé ces gouvernements à prendre des mesures d'austérité qui freinent la croissance économique et engendrent malheureusement des bouleversements sur le plan social, comme ce fut le cas dans des pays comme la Grèce et l'Espagne (Bance et Bernier, 2011). Ces mesures d'austérité entraînent une réduction du nombre de fonctionnaires et des budgets. Une fois de plus, cette situation laisse bien peu de place à l'amélioration des services gouvernementaux tout en augmentant la nécessité de faire plus avec moins, donc d'innover pour y parvenir.

Il arrive cependant que des innovations se produisent au sein des gouvernements. La nécessité d'innover et de transformer les gouvernements vient de pressions contradictoires. Les facteurs susmentionnés ont forcé les fonctionnaires à faire plus avec moins de ressources, mais les citoyens insatisfaits ou mieux formés en demandent encore plus à leur gouvernement. Sur une note plus positive, les nouvelles technologies rendent possibles de nouvelles solutions, autrefois impensables. Des personnes au sein de diverses organisations publiques tentent d'améliorer la prestation des services aux citoyens, leurs processus, leurs structures, etc. (Kernaghan, Marson et Borins, 2000). Lorsque les fonctionnaires trouvent suffisamment d'espace pour innover, ils le font.

Borins (1998 et 2008) et Riccucci (1995) ont écrit que l'innovation dans le secteur public était engendrée par des gestionnaires du type « héros obscur», qui agissent loin des sommets de la hiérarchie. Selon eux, l'espace nécessaire à l'autonomie croît de manière inver- 
sement proportionnelle à l'accumulation du pouvoir. Des îlots d'autonomie peuvent certes subsister (Skocpol et Finegold, 1982) et la capacité d'innover peut varier d'un secteur à l'autre (Considine et Lewis, 2007, p.582). Comme leurs études ont principalement été conduites aux États-Unis, où la relation avec le Congrès diffère de la tradition britannique, ils ont étudié la question de l'innovation de façon différente. Bien qu'on puisse prétendre que les innovations issues des États-Unis et du Canada se ressemblent (Borins, 2000), nous croyons qu'il est utile de tester l'incidence sur l'innovation du positionnement de l'appareil d'État dans un contexte canadien. Il est possible d'aller plus loin dans la thèse de ces auteurs et d'examiner les variations du taux d'innovation selon le positionnement de l'appareil d'État. Dans leur recherche qui employait également des théories américaines appliquées dans un contexte canadien, Bernier et Hafsi (2007) ont formulé l'hypothèse selon laquelle les entreprises d'État jouissent de plus d'autonomie sur le plan de l'innovation que les autres organisations du secteur public. Ces organisations, dont le fonctionnement se rapproche de celui du secteur privé, ont plus de chances de favoriser l'esprit d'entreprise et l'innovation en raison de la relation moins contraignante qu'entraîne la complexité de leurs activités principales et de l'autonomie que leur apportent les revenus générés par leurs activités commerciales. À partir d'une base de données portant sur toutes les candidatures reçues par les organisateurs du Prix IAPC pour gestion innovatrice ${ }^{1}$, il est possible de vérifier cette hypothèse.

Depuis 1990, année du lancement de ce prix canadien, inspiré par le modèle du Ash Institute de Harvard, près de 2000 candidatures ont été déposées. Grâce aux données, il est possible de se pencher sur les finalistes et les gagnants de chaque année. Les formulaires de candidature permettent le classement des candidatures selon les différents types d'organisations. Dans la première partie de cet article, on procédera à un survol de la documentation portant sur l'innovation avant d'aborder, dans la seconde partie, la question de l'utilisation du prix de l'IAPC comme échantillon représentatif de l'innovation. Enfin, on exposera la méthodologie de la présente étude et on proposera une conclusion tirée des résultats.

\section{L'INNOVATION DANS LE SECTEUR PUBLIC ET LES RÉCOMPENSES}

La littérature portant sur l'innovation dans le secteur public traite généralement des mécanismes internes des gouvernements (Albury, 2005; Crossan et Apaydin, 2010; Fernandez et Wise, 2010). Elle aborde le leadership dans le secteur public (Currie et autres, 2008), l'entrepreneuriat (Bernier et Hafsi, 2007), les dynamiques de groupe (Mohamed, 2002), les caractéristiques des employés innovateurs (Janssen, van de Vliert et West, 2004; Landau, 1993) ou des gestionnaires (Damanpour et Schneider, 2008). Le travail de Borins (2001) nous apprend que la plupart des innovations proviennent du personnel de l'organisation. C'est davantage le cas au Royaume-Uni et en Australie (82\%) qu'aux États-Unis (51\%). Walker (2012, p. 23), dans sa récente revue de la littérature portant sur l'innovation au sein des gouvernements locaux, conclut que « les antécédents internes à l'entreprise sont plus importants que ceux provenant de l'extérieur ${ }^{2}$ ». Il a également passé à la loupe les divers types d'innovations dans le secteur public, tels les processus (Walker, 2007). D'autres chercheurs (Bartunek et autres, 2007) ont examiné l'incidence des facteurs organisationnels sur les relations entre les structures organisationnelles et les dynamiques entre les groupes, de même que sur l'aspect routinier des innovations.

\footnotetext{
Voir www.cergo.enap.ca/CERGO/56/Innovation.enap

Toutes les citations dans cet article sont des traductions libres.
} 
Damanpour et Schneider (2008) ont pour leur part étudié la relation entre les caractéristiques des innovations et des gestionnaires et l'adoption des innovations dans les organisations publiques. Ils se sont basés sur des questionnaires administrés à des fonctionnaires locaux. En accord avec des idées liées au nouveau management public (NMP), les innovations visant l'amélioration des services ont été analysées (Albury, 2005; Walker, 2007). Les innovations semblent également avoir pour origine des réseaux de professionnels et de gestionnaires, mais elles nécessitent alors une adaptation (Considine et Lewis, 2007; Hartley, 2005). Une des approches de l'innovation est l'étude des besoins en efficacité (Osborne et Brown, 2005, p. 148). De plus, il a été observé que certaines organisations génèrent des innovations alors que d'autres les adoptent (Damanpour et Wischnevsky, 2006; Gow, 1992). Bien sûr certaines n'innovent pas du tout (Hartley, 2005). Lorsque les études ont été menées sur les innovations en réseaux et se sont penchées sur les liens unissant les organisations publiques entre elles, elles étaient généralement basées sur des études de cas (Arnaboldi, Azzone et Palermo, 2010; Denis et autres, 2002; Swan et Scarbrough, 2005), avec des possibilités limitées de généralisation. Crossan et Apaydin (2010) ont élaboré une méta-analyse qui lie le leadership, l'innovation en tant que processus et l'innovation en tant que résultat après une étude de centaines de documents publiés sur la question. Elles concluent tout d'abord qu'il existe une vaste gamme de variables dont on peut tenir compte et, ensuite, que de nombreuses perspectives théoriques sont prises en considération, ce qui rend la comparaison difficile. Elles suggèrent également de suivre Damanpour (1991), qui lui aussi a mené une méta-analyse. Selon lui, la structure et la position sont des déterminants importants de l'innovation. Cette importante étude est cependant limitée, car aucune proposition ne relie les éléments. Parmi les grilles théoriques employées, l'action sociale jouit d'une place de choix (Hellström, 2004). Récemment, un intérêt a de nouveau été porté sur les effets de l'environnement (Osborne et Brown, 2011, p. 1342), mais il manque toujours des travaux portant sur le positionnement de l'appareil d'État. Comme l'écrivent Considine et Lewis (2007, p. 583), bien que l'innovation soit perçue comme le résultat d'interactions entre politiciens et fonctionnaires, elle est façonnée par les structures institutionnelles qui les abritent. On considère en général que la spécialisation a un effet positif sur l'innovation ainsi que la différenciation fonctionnelle et le professionnalisme et, d'un autre côté, on tient pour acquis que la centralisation pourrait avoir des effets négatifs (Damanpour, 1991, p. 558).

En examinant les études d'auteurs qui se sont penchés sur les effets de l'environnement sur l'innovation, on constate une relation en U entre la décentralisation et l'innovation dans le secteur public (Nohria et Gulati, 1996). L'innovation se produit dans un type particulier d'espace institutionnel (Considine et Lewis, 2007) ou dans des systèmes ouverts (Osborne et Brown, 2011, p. 1343). Damanpour et Schneider (2008) ont souligné l'importance de l'urbanisation et de l'accroissement de la population. Dougherty et Dunne (2011) proposent un modèle préliminaire pour l'organisation de l'écologie des innovations complexes, mais dans le secteur privé, comme ce fut le cas du travail souvent cité de Damanpour (1991) portant sur l'innovation. Pour eux, l'innovation est engendrée par l'écologie des contacts entre de multiples organisations, institutions et autres intervenants tant du secteur privé que public, où des savoirs sont créés, combinés et recombinés. La vision selon laquelle l'environnement peut favoriser l'esprit d'innovation est intéressante (Miller, 1998). L'innovation dans le secteur public pourrait être engendrée par l'organisation, mais également par les changements environnementaux, tels que les attentes plus élevées des citoyens et leurs configurations changeantes (Albury, 2005; Lekhi, 2007; Walker, 2012, p. 3). Borins (1995) a étudié l'incidence des contraintes financières sur les gouvernements, l'accessibilité aux technologies de l'information et la diversification du personnel dans une étude de 
18 cas. Il apparaît qu'il faudrait inclure les facteurs environnementaux dans plus de recherches sur la question (Damanpour et Schneider, 2008, p. 514-515).

Une faible portion des recherches portant sur l'innovation dans le secteur public a eu pour objet les prix pour l'innovation dans le secteur public à un moment où ce secteur devait être réinventé. L'idée de prix récompensant l'innovation a émergé à la fin des années 1980. En accordant un tel prix, on récompensait le travail bien fait. Les prix ont également servi de vecteur pour rendre publiques les innovations, grandes ou petites, qui méritaient d'être adoptées dans d'autres contextes (Hartley et Downe, 2007). Un autre effet possible de la mise en candidature pour ces prix pouvait être l'occasion de rétroaction, la création de réseaux d'innovateurs et la possibilité de faire connaître certaines idées sur l'innovation et les manières de les reproduire (Gow, 1992, p. 4; Walker, 2007, p. 594). Les prix sont devenus une façon de populariser des solutions nouvelles et intéressantes aux problèmes auxquels faisaient face de nombreuses organisations du secteur public. Certains des créateurs de ces prix espéraient également qu'ils aideraient à améliorer le moral des fonctionnaires (Borins, 2000). Ils s'attendaient aussi à ce que l'image du secteur public soit redorée par la diffusion des pratiques exemplaires (Galimberti, 2003; Moore, 2005). Les prix existaient auparavant, mais pour d'autres motifs, par exemple la performance. La nouveauté résidait dans l'accent mis sur l'innovation, dans l'admission du fait qu'au cours d'une période de turbulences, il fallait transformer les organisations publiques et que ces améliorations devaient être récompensées (Kernaghan, Brian et Borins, 2000).

\section{LE PRIX INNOVATION AU CANADA}

C'est aux États-Unis que les premiers prix pour l'innovation ont été lancés. Le tout premier programme, créé en 1985 à l'Université Harvard, est géré par l'Ash Institute de la Kennedy School; il offre des récompenses financées par la Ford Foundation dans de multiples catégories (Moore, 2005). Pour souligner son 20e anniversaire, un ouvrage a été publié sous le titre Innovations in Government (Borins, 2008) afin d'offrir une synthèse des approches émergentes. La capacité du prix à stimuler l'innovation a été reconnue et les programmes de prix se sont multipliés dans le monde (Borins et Walker, 2012). Le Canada a suivi l'exemple américain.

Les candidatures à ces prix sont de bons échantillons pour l'étude des pratiques exemplaires dans l'administration publique (Borins, 2008). Elles peuvent être considérées comme de bons indicateurs pour d'éventuelles études à grande échelle de l'innovation dans le secteur public. " Les prix sur l'innovation dans le domaine de la gestion publique ne récompensent pas des idées neuves n'ayant pas fait leurs preuves : ils choisissent les meilleures candidatures sur la base des résultats, de la reproductibilité des expériences et de leur originalité » (Borins, 2000, p. 47). On a souvent fait référence à ces prix dans des publications sur l'innovation (Borins, 2008; Galimberti, 2003). Cela a été le cas au Canada (Borins, 2000; Gow, 1992; Glor, 1998) et d'autres ont fait de même pour des prix aux États-Unis (Borins, 2001 et 2008; Golden, 1990; Rangarajan, 2008). On pourrait soutenir que les candidatures à de tels prix représentent non pas des innovations comme telles, mais des indicateurs d'innovation. Il faut également accepter l'hypothèse que les finalistes et les gagnants peuvent être considérés comme des innovateurs pour pouvoir utiliser ces données comme un échantillon de recherche.

Les prix pour l'innovation ont été introduits au Canada en 1990 au cours des heures de gloire du NMP, lorsque l'accent a été mis sur les pratiques exemplaires. En laissant de côté 
ses aspects idéologiques (Metcalfe, 1993), le mouvement du NMP avait pour objectif d'engendrer une plus grande efficacité dans le secteur public et a été reconnu comme tel, bien que ses limites soient désormais reconnues (Bernier et Angers, 2010; Dunleavy et autres, 2006). L'un des bienfaits de l'avènement du NMP, cependant, est que le mouvement ait lancé le débat sur les possibles innovations dans le secteur public. Borins (2008) a été plus loin que la proposition de "réinventer le gouvernement " selon laquelle dans un secteur public agité, l'innovation devient une nécessité et qu'il faut réinventer l'État. C'était vrai au Canada dans les années 1980 et 1990 où le moral des fonctionnaires avait besoin d'un remontant. Les prix ont joué ce rôle (Borins, 2000). Pour Borins, l'innovation est une nécessité permanente pour que l'État puisse continuer à servir la société. Il a aussi été dit que les candidatures doivent généralement être approuvées par un haut fonctionnaire ou un politicien (Osborne et Brown, 2011, p. 1340). Cela pouvait représenter une limite à la véritable innovation, mais puisque la vague d'innovation constituait une réponse au besoin urgent des gouvernements de "faire plus avec moins ", on peut argumenter que cela tient plutôt lieu à une institutionnalisation des processus d'innovation, ce qui lui confère légitimité et reconnaissance.

Le système politique canadien est un laboratoire intéressant pour cette étude. Le gouvernement fédéral et les dix provinces fonctionnent avec le même système politique hérité de la tradition parlementaire britannique, mais ont développé des cultures politiques différentes (Bernier, Brownsey et Howlett, 2005). Le système fédéral a été conçu pour prendre en compte l'existence de deux importants groupes linguistiques et pour s'adapter à la géographie. Les Canadiens jouissent d'un système politique stable. Au cours des 20 dernières années, on a assisté à une rotation des partis dans toutes les régions - exception faite de l'Alberta -, et les partis conservateurs, libéraux et NPD se sont succédé au pouvoir. La croissance démographique a été importante et, comme dans le reste du monde industrialisé, les 20 années dernières ont été agitées, en raison de l'obligation de gérer des déficits et de la nécessité de s'adapter aux changements touchant l'économie mondiale. La recherche empirique dans le secteur public au Canada a généralement fourni des études qualitatives détaillées. Celle de Glor (2002), par exemple, explore la stratégie du gouvernement Blakeney en Saskatchewan, mais ne permet toutefois pas les généralisations.

Toutes les administrations publiques canadiennes sont admissibles à ces prix de l'innovation. Chaque année depuis 1990, de 60 à 100 organisations issues des municipalités, des provinces, des territoires ou du gouvernement fédéral ont posé leur candidature. Comme aux États-Unis, on sollicite les candidatures par lettres et par courriels (Galimberti, 2003; Golden, 1990). Certes, il est possible de remettre en question le caractère novateur des candidatures, mais ces dernières demeurent les meilleurs indicateurs d'innovation disponibles, puisqu'elles font l'objet d'une promotion et sont approuvées par les fonctionnaires des organisations publiques. Pour le prix de l'IAPC (Prix IAPC pour gestion innovatrice), les candidatures sont habituellement d'abord présentées lors de compétitions régionales ou dans le cadre de forums plus spécialisés. Il est intéressant de noter que les meilleures candidatures, qu'on pourrait considérer comme des innovations, sont souvent copiées (Borins, 2000, p. 68).

Un nombre restreint d'études sur l'innovation dans le secteur public au Canada ont été conduites. On remarque en particulier le travail de Borins et ceux de Gow (1992) et de Glor (1998). Ils ont étudié certains cas intéressants d'innovation au Canada de divers gouvernements en employant des méthodes de sondage et des interviews auprès de différents groupes dans la communauté de l'administration publique afin d'élaborer des théories sur 
l'innovation dans le secteur public. Gow (1994, p. 14) était plus intéressé par le processus d'innovation que par ses déterminants. Sa perspective est plus vaste que celle des candidatures pour les prix sur l'innovation. Il était intéressé, par exemple, par l'introduction de la rationalisation des choix budgétaires (planification, programmation, préparation du budget), puis par le budget base zéro, par l'arrivée du principe de l'ombudsman, etc. Les cas qu'il a étudiés couvrent une période s'étendant de la fin des années 1960 au début des années 1980, ce qui représente une ère différente pour l'administration publique canadienne. L'exploration qu'a réalisée Glor (2002) des processus d'innovation au sein du gouvernement de la Saskatchewan au cours de la période 1971-1982 est particulièrement intéressante puisque c'est de la Saskatchewan qu'est venue l'assurance maladie au Canada, ainsi que d'autres importantes réformes sociales canadiennes. Le gouvernement de la Saskatchewan s'est longtemps montré innovateur, et il est surprenant de constater que cet aspect n'est pas reflété dans les données couvrant les périodes subséquentes.

Glor (2002, p. 155) a étudié les mises en candidature pour les prix de 1990 à 1999, en se penchant sur les médaillés et les finalistes. Ses résultats indiquent que la Saskatchewan, sujet du livre, n'a posé que 25 fois sa candidature au cours de la décennie et s'est contentée d'un second prix et d'un finaliste. Glor (2002, p. 172 et 178) souhaitait généralement montrer que la volonté et la détermination peuvent être des stratégies puissantes pour l'innovation. Nous reconnaissons ce fait mais dans notre étude, nous examinons la totalité de la population des candidatures pour le Prix IAPC sur une période de 20 ans. Notre recherche systématique de relations, avec l'introduction de variables originales et non disponibles par l'IAPC, n'a jamais été réalisée au Canada. Les cas sont importants et fournissent des études approfondies. Nous proposons un aperçu plus vaste qui pourrait éventuellement être renforcé par des études de cas, pour une meilleure généralisation.

\section{VUE D’ENSEMBLE DE L'ÉTUDE ET MÉTHODOLOGIE}

Cette étude emploie comme base de données les candidatures au Prix IAPC pour gestion innovatrice soumises par les organisations fédérales et provinciales du Canada ${ }^{3}$. Nous avons compilé les données provenant de toutes les candidatures depuis son lancement en 1990. Les candidatures sont disponibles sous forme électronique pour les années 2000, ce qui nous permet d'étudier tous les aspects de l'innovation dans le secteur public. Comme la base de données couvre une longue période, il nous est également possible d'étudier les mécanismes à l'œuvre sur le long terme. Cette base de données, la première de ce type au Canada, a été rendue accessible au public en 2013. Comme il en a été question plus haut, elle peut servir de base à une discussion sur les tendances actuelles dans l'innovation au sein de l'administration publique, et des analyses statistiques pourraient être reproduites.

La base de données a été divisée en trois groupes : les 1998 candidatures présentées entre 1990 et 2011, l'ensemble des sélections annuelles variant de 6 à 10 finalistes (soit un total de 162 candidatures sélectionnées) ainsi que les trois gagnants invités chaque année à

3 Un lecteur critique a soulevé deux importantes limitations liées à la source des données. D’abord, certaines organisations très innovantes pourraient, pour une raison ou une autre, ne pas être motivées à soumettre des candidatures. Les raisons menant à cette décision sont multiples : les organisations pourraient être déjà reconnues de différentes manières pour leur excellente performance, les gestionnaires pourraient ne pas se considérer comme des fonctionnaires, etc. Ensuite, seules les organisations proposant des innovations ayant connu du succès sont incluses. Les organisations dont les innovations ont connu l'échec ou qui n'ont pas montré d'efforts pour innover disparaissent. Résultat, nos recherches devront être considérées avec ces objections en tête. 
présenter leur projet à la conférence annuelle de l'IAPC. Le dépôt d'une candidature signifie que le candidat croit avoir accompli quelque chose d'innovateur. Ainsi, non seulement les trois gagnants, mais tous les finalistes sélectionnés sont vus comme des innovateurs couronnés de succès par un jury constitué principalement de spécialistes d'expérience et comprenant, certaines années, un universitaire (Galimberti, 2003). Les candidatures suggèrent le développement d'une culture de l'innovation dans le secteur public canadien. Les fonctionnaires semblent valoriser l'innovation, ils songent à poser leur candidature et remportent parfois un prix. Ce concours de l'IAPC est l'étape ultime d'un processus de sélection par lequel les organisations doivent passer après avoir remporté des concours locaux ou spécialisés.

Parmi les 1998 candidatures soumises depuis la mise sur pied des prix en 1990 et jusqu'à 2012, 396 provenaient de municipalités et quelques-unes étaient issues de partenariats d'organisations appartenant à différents gouvernements. Ces candidatures ne sont pas prises en compte dans la présente étude, principalement en raison du manque de données sur les partis politiques et l'évolution démographique au palier municipal. Le problème est aggravé par la vague de fusions qui a déferlé sur un grand nombre de villes. Le palier municipal est cependant bien couvert par d'autres études sur l'innovation (Hartley et Downe, 2007; Walker, Damanpour et Devece, 2010). Dans notre analyse, les trois territoires du nord (les plus petits gouvernements) ont également été exclus pour deux raisons : un nombre insuffisant d'innovations ont été soumises par l'ensemble des territoires (en tout, un total de 27 soumissions en 20 ans) et la Loi sur le Nunavut ( $1{ }^{\mathrm{er}}$ avril 1999), qui séparait le Nunavut des Territoires du Nord-Ouest, ne permet pas une analyse significative des données historiques. Par conséquent, il demeure dans le cadre de notre étude 11 gouvernements, le fédéral et les 10 provinces, avec un total de 1602 candidatures et 146 finalistes représentant 484 organisations. Nous entreprenons notre analyse par une rapide description de certaines tendances dans les données, suivie d'une analyse de régression plus élaborée.

Comme nous l'avons mentionné dans l'introduction, il est possible de classer les organisations gouvernementales en trois grandes catégories. Elles sont regroupées ici aux fins d'analyse en quatre ensembles liés à leur degré d'autonomie et de contrôle politique. Il y a en premier lieu les organismes centraux qui gouvernent l'appareil administratif : le Bureau du Conseil privé à Ottawa ou le ministère du Conseil exécutif au Québec, les secrétariats des cabinets ailleurs, le Conseil du Trésor, les bureaux ou ministères des relations entre le fédéral et les provinces, ou les bureaux ou ministères des relations intergouvernementales, la Commission de la fonction publique ou le Bureau du premier ministre (Dunn, 2010; Savoie, 1999; White, 2005). Dans la seconde catégorie, on trouve les ministères traditionnels tel le ministère de l'Éducation ou de l'Agriculture. Certains ministères comme celui des Finances sont parfois considérés comme des organisations centrales parce que leur travail influence les autres ministères, mais dans notre classification, les ministères des Finances font partie des autres ministères. On pourrait créer une classification faisant la distinction entre les ministères horizontaux tels que les Finances et les verticaux comme dans les exemples précédents. Nous avons procédé à des tests où ces ministères horizontaux étaient classifiés avec les organismes centraux et nous n'avons pas noté de variations dans les résultats. Dans un ouvrage classique, Kernaghan et Siegel (1999, p. 202) emploient la définition de Hodgetts : «Un ministère est une unité administrative comprenant une ou des composantes organisationnelles sur lesquelles un ministre détient la gestion directe ou le contrôle. » Cela limite la définition aux portefeuilles ministériels majeurs auxquels des responsabilités sont assi- 
gnées. Les ministères sont, depuis 1840, les organisations qui forment le cœur de l'administration publique canadienne (Tardi, 2010).

Troisièmement, on trouve les organisations plus autonomes, les conseils et les commissions qui sont créés pour veiller à l'implantation de politiques (Richmond et Siegel, 1994), mais dont les formes sont variées (Pollitt et autres, 2004, p. 245) au Canada comme ailleurs. Même si la prestation des services s'est améliorée au Canada (Zussman, 2010), une longue liste de ces organisations existe aux paliers fédéral, provincial et municipal. De telles organisations jouissent habituellement d'un certain degré d'autonomie face au gouvernement, elles sont dotées d'un conseil d'administration distinct, mettent en œuvre un nombre limité de fonctions de type gouvernemental et n'ont pas le pouvoir de percevoir des taxes, mais reçoivent leur financement du gouvernement (Richmond et Siegel, 1994, p. 7). Elles sont chargées de missions spécifiques et dégagées de supervision politique pour leurs activités routinières. Par exemple, Passeport Canada n'est pas, en théorie, lié à la moindre question de politique publique. Le degré d'autonomie de ces organisations varie en fonction de la nature de leur mission et en fonction de la proportion de leurs revenus qui sont liés à leur travail. Leurs activités peuvent ou non inclure un certain pouvoir de réglementation. Le Conseil de la radiodiffusion et des télécommunications canadiennes (CRTC) a par exemple la capacité de réglementer les télécommunications. Les dénominations des organisations autonomes sont variées : bureaux, conseils, commissions, compagnies...

Parmi ces organisations autonomes, on distingue une quatrième catégorie, les sociétés d'État, ou sociétés de la Couronne comme on les appelle au Canada, qui sont des organisations hybrides dotées d'activités commerciales et d'objectifs politiques. Cette catégorie pourrait être considérée comme un autre type d'organisation autonome, mais compte tenu de la liberté additionnelle dont elles jouissent grâce à leur capacité à profiter de sources de revenus indépendantes, ces sociétés méritent d'être considérées à part (Bernier et Farinas, 2010). Les sociétés de la Couronne varient grandement en importance. Hydro-Québec détient par exemple plus de 55 milliards en actifs et compte sur des revenus de 12 milliards de dollars, alors que la Société des établissements de plein air du Québec ne rapporte qu'un profit net de 3,5 millions. Ces sociétés ont été importantes dans l'histoire du Canada, car l'État avait une capacité limitée de régulation de l'économie (Roberts, 2010).

\section{TABLEAU 1 : SOMMAIRE DES ORGANISATIONS GOUVERNEMENTALES}

\begin{tabular}{|c|c|c|}
\hline Catégorie & Description & Exemple \\
\hline Organismes centraux & $\begin{array}{l}\text { Coordination des activités de tout } \\
\text { l'appareil administratif }\end{array}$ & Conseil privé, Conseil du Trésor \\
\hline Ministères & $\begin{array}{l}\text { Tous les ministères jouissant d'un } \\
\text { portefeuille }\end{array}$ & $\begin{array}{l}\text { Ministère de la Santé, ministère } \\
\text { des Finances }\end{array}$ \\
\hline Organismes autonomes & $\begin{array}{l}\text { Organismes dotés de missions } \\
\text { spécifiques, leurs activités peuvent } \\
\text { inclure le pouvoir de réglementer }\end{array}$ & $\begin{array}{l}\text { CRTC, Agence spatiale } \\
\text { canadienne, Conseil des arts }\end{array}$ \\
\hline Sociétés d’État & $\begin{array}{l}\text { Organisations dont la mission est de } \\
\text { type commercial et dont le principal } \\
\text { actionnaire est le gouvernement }\end{array}$ & $\begin{array}{l}\text { Hydro-Québec, Société des } \\
\text { alcools du Québec, Postes } \\
\text { Canada }\end{array}$ \\
\hline
\end{tabular}




\section{L'hypothèse}

Selon nous, l'autonomie des organisations gouvernementales est un facteur qui favorise la capacité des organisations à innover. Cependant, compte tenu des données dont nous disposons, nous avons travaillé sur une hypothèse légèrement différente qui met plutôt l'accent sur le nombre de candidatures présentées au Prix IAPC comme une représentation de l'innovation. Ainsi, notre principale hypothèse est la suivante : les organisations autonomes innovent plus que celles qui jouissent de moins d'autonomie.

C'est là une hypothèse simple qui reflète un concept central de l'administration publique, c'est-à-dire que le fait d'accorder de l'autonomie aux gestionnaires et aux organisations leur permet d'innover, de créer des processus plus efficaces et efficients et, au final, de générer de meilleurs résultats. L'innovation et la créativité sont depuis longtemps associées à la liberté et à l'autonomie. La souplesse de supervision favorise l'innovation et la créativité dans les organisations publiques. Les héros obscurs pourraient avoir eu plus d'espace pour manœuvrer (Borins, 1998; Riccucci, 1995). Cela reflète les relations attendues décrites par Damanpour (1991). Les organisations centrales des gouvernements subissent de fortes pressions politiques, elles sont les moins autonomes de toutes les administrations publiques (Bernier, Brownsey et Howlett, 2005). Tout changement, ou innovation, affronte des barrières législatives et même constitutionnelles avant sa mise en œuvre, ce qui réduit le nombre possible d'innovations générées par les organismes centraux. Nous émettons l'hypothèse selon laquelle le degré d'autonomie augmente lorsqu'une organisation se trouve plus loin du cœur du gouvernement. Les ministères sont situés entre les deux. Ils doivent rendre des comptes à la Chambre des communes. À l'autre extrémité du spectre, lorsque les sociétés d'État font des profits, les gouvernements perdent un peu le contrôle sur elles.

\section{Les données, la méthode, le modèle et les résultats}

Le tableau 2 offre un survol des 484 organisations ayant soumis des candidatures au Prix IAPC au cours des 22 dernières années. Au total, 1602 candidatures ont été déposées. Le nombre de candidatures présenté dans le tableau est plus élevé (1 629 candidatures), car certaines candidatures étaient des partenariats formés d'organisations appartenant à différentes catégories.

\section{TABLEAU 2: LES CANDIDATURES ET LES FINALISTES SELON LES DIFFÉRENTS TYPES D'ORGANISATIONS ADMINISTRATIVES}

\begin{tabular}{|l|c|c|c|c|}
\hline & $\begin{array}{c}\text { Nombre } \\
\text { d'organisations }\end{array}$ & $\begin{array}{c}\text { Nombre de } \\
\text { candidatures }\end{array}$ & $\begin{array}{c}\text { Nombre de } \\
\text { finalistes }\end{array}$ & $\begin{array}{c}\text { Pourcentage } \\
\text { des finalistes }\end{array}$ \\
\hline Organismes centraux & 36 & 107 & 12 & $11,21 \%$ \\
\hline Ministères & 186 & 1069 & 90 & $8,42 \%$ \\
\hline Organismes autonomes & 196 & 341 & 41 & $12,02 \%$ \\
\hline Sociétés d'État & 66 & 112 & 9 & $8,04 \%$ \\
\hline Total & $\mathbf{4 8 4}$ & $\mathbf{1 6 2 9}$ & $\mathbf{1 5 2}$ & $\mathbf{9 , 3 3 \%}$ \\
\hline
\end{tabular}


À la vue de ce tableau, on constate d'abord qu'un nombre plutôt élevé de candidatures $(65,6 \%)$ provient des ministères. Ces derniers forment la colonne vertébrale des fonctions gouvernementales et auront plus tendance à chercher des prix liés à l'administration publique. Certains ministères ont présenté de nombreuses candidatures au cours des ans, comme Développement des ressources humaines Canada (63 candidatures), Travaux publics et Services gouvernementaux Canada (46 candidatures), le ministère des Ressources naturelles de l'Ontario ( 40 candidatures) et le ministère des Transports de l'Ontario (38 candidatures). Bien que certains ministères présentent plusieurs candidatures, ils ne remportent pas souvent le Prix. Ainsi, malgré ses 63 candidatures, Développement des ressources humaines Canada n'a obtenu qu'un prix et n'a été sélectionné que quatre fois parmi les finalistes, soit un ratio de $6,3 \%$ de finalistes/candidatures, ce qui est inférieur à la moyenne des autres ministères $(8,4 \%)$ et de celle des organisations dans leur ensemble $(9,3 \%)$. Tous les ministères ne souffrent pas d'une feuille de route aussi faible. L'organisation ayant connu le plus de succès au Prix de l'IAPC (en nombre de prix remportés) est le ministère du Développement économique, du Commerce et de l'Emploi de l'Ontario qui a remporté un prix à 4 reprises ( 3 de bronze et un d'or) et a été finaliste 6 fois en 24 candidatures. Cela représente un taux de succès de $25 \%$, de loin supérieur à celui des autres organisations. Cependant, un tel résultat n'est qu'anecdotique et ne tient pas compte de l'ensemble de la situation. Certaines organisations n'ont soumis qu'une seule candidature en 22 ans, mais sont rentrées à la maison avec un trophée. Revenu Québec, ministère récemment transformé en organisme, a remporté l'or en 2009 et en 2012.

Les données du tableau 2 indiquent que les ministères ont relativement peu de succès au Prix IAPC, si l'on considère qu'ils représentent la majorité des candidatures. Ils ne font cependant pas plus mal que les sociétés d'État qui non seulement ne gagnent pratiquement jamais, mais en plus ne soumettent que très rarement leur candidature. Ces sociétés ont soumis en moyenne 1,7 candidature par organisation au cours des 22 dernières années. Avec un nombre si limité de candidatures, on s'attendrait à ce que ces dernières soient sans doute exceptionnelles, ou à tout le moins dignes de mention. Cependant, seules 9 des 112 candidatures ont atteint le stade de finalistes et 3 ont gagné, ce qui représente à peu près le ratio de gagnants/finalistes au Prix IAPC. L'organisation la plus victorieuse de cette catégorie est sans doute SaskTel avec trois candidatures dont deux ont été retenues. L'une de celles-ci a remporté un trophée d'argent. Il faut également mentionner BC Hydro, la seule société de la Couronne à avoir remporté l'or, soit en 1991, pour une réorganisation qui améliorait la planification stratégique en passant à une structure aplatie fondée sur les unités d'affaires. Bien que cette innovation ait été reconnue par l'IAPC, elle n'a pas amélioré la capacité de cette société à innover: BC Hydro a depuis présenté cinq autres candidatures, mais aucune n'a reçu de mention du jury. Il est possible que ça ne soit pas la localisation au sein de l'appareil d'État qui compte, mais plutôt une distance suffisante par rapport au sommet d'un ministère.

Les deux dernières catégories, les organismes centraux et les organismes autonomes, se situent clairement au-dessus de la moyenne en ce qui concerne le ratio finalistes/candidatures. Les organismes centraux ne jouissent peut-être pas de beaucoup d'autonomie, mais ils continuent de se porter candidats plus souvent (avec une moyenne de presque 3 candidatures par organisation) et mieux (avec 12 finalistes sélectionnés parmi 107 candidatures, un ratio de $11,21 \%$ ) que les autres types d'organisations. Il est également possible qu'ils échappent aux règles strictes qu'ils imposent aux autres organisations. Dans cette catégorie, on compte un bon nombre d'organisations telles que le ministère de l'Exécutif des Territoires du NordOuest et le Conseil des sous-ministres de l'Alberta qui n'ont soumis qu'une seule candidature 
et ont remporté le prix. La première a obtenu la seconde place pour un projet intégrant des prestations en éducation, santé et services auprès de la communauté tlicho, tandis que la seconde a remporté le premier prix pour sa stratégie de développement des ressources humaines qui veillait à ce que la fonction publique de l'Alberta soit bien préparée pour faire face à ses futurs besoins en personnel. Les deux innovations ont été reconnues, mais les organisations ont soit choisi de ne plus poser de candidature ou n'ont jamais plus senti qu'elles avaient en main des innovations dignes d'être primées par le jury. À l'autre bout de l'horizon, le Secrétariat du Conseil de gestion de l'Ontario a soumis 29 candidatures à l'IAPC, mais une seule s'est retrouvée parmi les finalistes.

Cela nous conduit aux organismes autonomes, lesquels sont responsables de missions particulières sans trop de supervision politique. Ils présentent une moyenne de 1,75 innovation par organisation, avec un taux de succès de $12 \%$ plus élevé que toutes les autres catégories et presque $50 \%$ plus élevé que celui des sociétés de la Couronne. D’une part, deux organismes autonomes ont posé leur candidature plus de 10 fois et ont affiché des résultats mitigés : l'Agence de revenu du Canada avec 25 candidatures et 2 finalistes et le Service correctionnel du Canada avec 15 candidatures sans la moindre reconnaissance du jury. D'autre part, la seule organisation à avoir remporté deux trophées d'or demeure Revenu Québec, victorieux en 2009 et 2012, soit avant et après être passé au statut d'organisme autonome en 2010-2011. Il serait intéressant de se pencher sur cette organisation pour obtenir une compréhension de sa culture de l'innovation. Ses deux précédentes candidatures, en 2005 et 1994, ne s'étaient pas rendues au stade de finalistes. La question de savoir si le changement de ministère à organisme autonome a permis à Revenu Québec de devenir un leader de l'innovation dans les services publics mériterait sans doute sa propre étude de cas.

Bien que l'information qui précède soit anecdotique, les données disponibles confirment la relation entre les différents types d'organisations publiques et la qualité de l'innovation, telle qu'elle est évaluée par le jury du Prix IAPC au cours des ans. Le tableau suivant présente une régression des probits portant sur les 1602 candidatures au Prix IAPC pour gestion innovatrice en employant Stata v12.1. Avec cette régression, nous tentons de découvrir si le type d'une organisation a une influence sur les candidatures retenues parmi les finalistes. La variable dépendante est dichotomique : «1» pour les candidatures retenues parmi les finalistes, « 0 » pour toutes les autres. OrganismesCentraux, Ministères, OrganismesAutonomes et SociétésCouronne sont également des variables dichotomiques employées pour déterminer si une candidature a été soumise par un type d'organisation. Nous utilisons des variables séparées parce qu'un sous-ensemble de candidatures ont été soumises en partenariat par plus d'une organisation, ce qui fait que certaines candidatures se trouvent dans plus d'une catégorie. CandidaturesAnnée est une variable de contrôle : plus il y a de candidatures pour une année, moins il y a de chances que les organisations soient sélectionnées. Pendant 21 ans, l'IAPC a déterminé un thème annuel pour son Prix, engendrant alors de vastes fluctuations du nombre de candidatures chaque année, mais les finalistes ont généralement été au nombre de 7 à 9. La régression donne des résultats similaires sans ce contrôle, mais nous avons cru important de prendre en compte la différence dans le nombre de candidatures. Nous voulions également établir un contrôle pour le nombre de finalistes sélectionnés chaque année, mais cette variable s'est révélée non significative et a été rejetée. 


\section{TABLEAU 3 : RÉGRESSION DES PROBITS}

Logarithme du rapport de vraisemblance $=-472,90003$

Nombre de candidatures $=1602$

LR chi2(5) $=31,93$

Prob $>$ chi $2=0,0000$

Pseudo R2 $=0,0327$

\begin{tabular}{l|c|c|c|c} 
Finaliste & Coefficient & Erreur type & Z & P>Z \\
\hline OrganismesCentraux &, 4727204 &, 2891894 & 1,63 & 0,102 \\
\hline Ministères &, 436248 &, 2848625 & 1,53 & 0,126 \\
\hline OrganismesAutonomes &, 5971302 &, 2800299 & 2,13 & 0,033 \\
\hline SociétésCouronne &, 3707767 &, 3268678 & 1,13 & 0,257 \\
CandidaturesAnnée &,- 0081907 &, 00168 & $-4,88$ & 0,000 \\
Constante & $-1,058459$ &, 3221154 & $-3,29$ & 0,001 \\
\hline
\end{tabular}

En plus du contrôle de la variable CandidaturesAnnée, la régression ne comporte qu'une variable significative, OrganismesAutonomes, qui indique si un organisme autonome a participé à la réalisation de l'innovation. La relation est positive. Ainsi, les organismes autonomes ont tendance à atteindre le stade de finalistes plus souvent que les autres organisations publiques. Les organismes centraux obtiennent un résultat semblable, mais il n'est significatif qu'au niveau $\mathrm{p}<, 10$, ce qui n'est pas assez important pour être considéré comme différent de 0 . Les résultats des ministères et des sociétés de la Couronne ne sont pas significatifs.

Pour tester davantage notre hypothèse, nous avons voulu déterminer si l'emploi de la variable dichotomique Finalistes était suffisant pour évaluer la qualité des innovations mises en candidature. En remplaçant cette variable par une autre intitulée Prix, nous voulions capturer une image plus vaste de la qualité de chaque innovation telle qu'elle est évaluée par le jury. Prix est une échelle ordonnée avec les valeurs suivantes : 0 : aucune reconnaissance; 1 : finalistes; 2 : prix de bronze; 3 : prix d'argent; 4 : prix d'or. Les résultats de la régression ordonnée des probits sont présentés dans le tableau ci-dessous. Les variables indépendantes demeurent inchangées. 


\section{TABLEAU 4 : RÉGRESSION ORDONNÉE DES PROBITS}

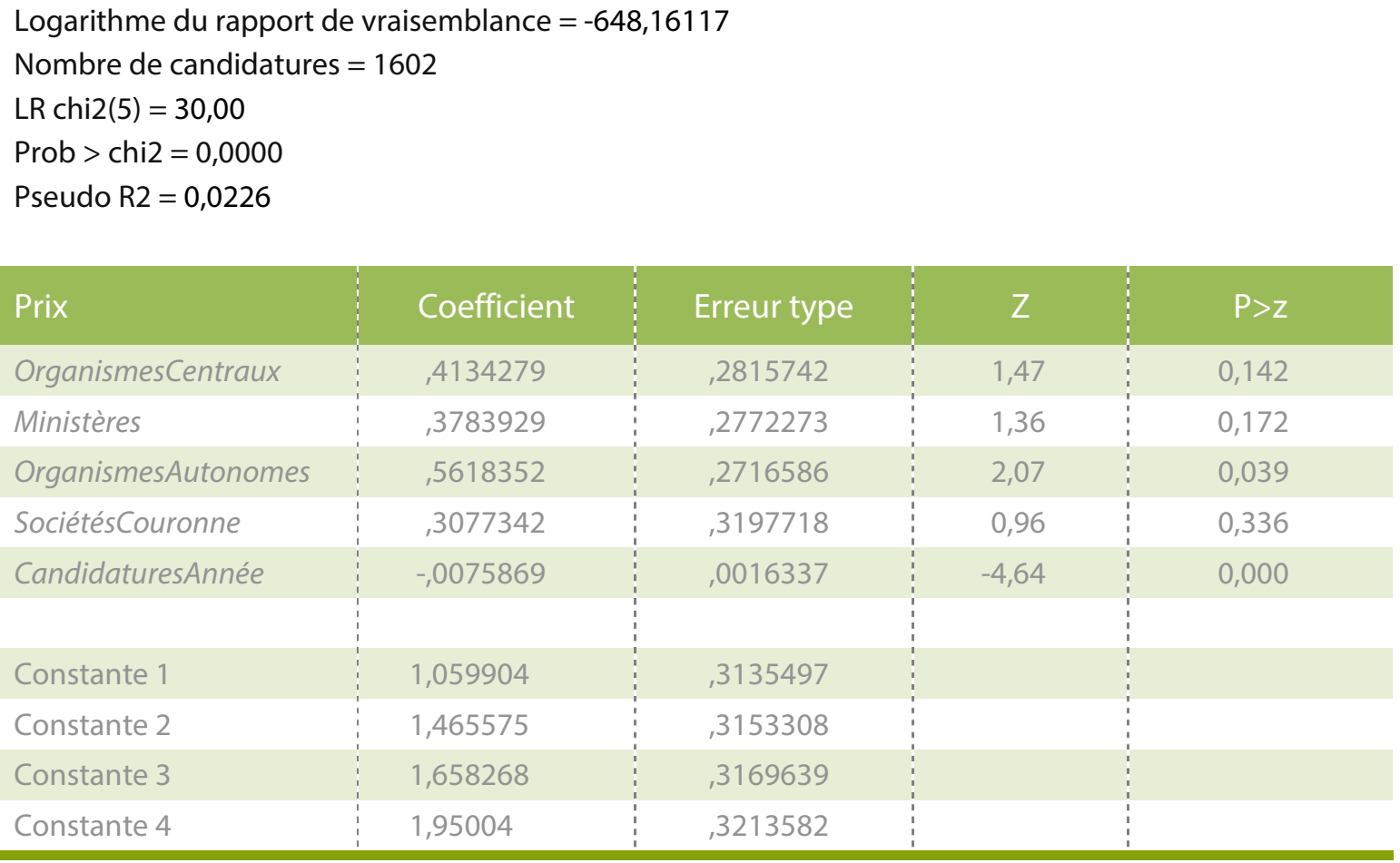

Les résultats de cette seconde régression sont pratiquement identiques à ceux de la première. Une fois encore, une seule relation semble significative : les organismes autonomes remportent plus de prix que tous les autres types d'organisations publiques. La constance de ces résultats soutient le postulat selon lequel les candidatures retenues sont révélatrices de la qualité de l'innovation. Ceci est important parce que les juges qui sélectionnent les candidatures ne sont pas les mêmes que ceux qui déterminent qui remporte un prix. Selon nos résultats, cependant, les deux groupes d'évaluateurs sont plus impressionnés par les candidatures provenant d'organisations autonomes que par celles soumises par les autres organisations publiques.

Les candidatures ne sont que des indicateurs, possiblement les meilleurs compte tenu des données disponibles, mais il faut garder en tête quelques limitations ${ }^{4}$. Par exemple, les projets des finalistes figurant sur la liste des sélectionnés pourraient être considérés comme des cas de brillantes innovations, mais on ne peut en avoir la certitude. Les trois gagnants sont considérés comme étant supérieurs, mais il serait intéressant d'observer, année après année, en quoi consiste l'écart dans l'esprit du jury entre les vainqueurs et les autres finalistes. Par ailleurs, des projets très intéressants peuvent n'avoir pas fait l'objet de candidatures, et la qualité pourrait varier de manière considérable d'une année à l'autre. De plus, les membres du jury varient, comme c'est le cas des différentes préférences quant à l'équilibre à atteindre entre les provinces et le gouvernement fédéral, par exemple. De toute évidence, le personnel des sociétés d'État soumet rarement des candidatures à ce concours.

4 Encore une fois ici, les commentaires d'un de nos lecteurs critiques ont été d'un grand secours. Nous le remercions. 


\section{DISCUSSION ET CONCLUSION}

Est-il possible de conclure sur la base de ces données que l'autonomie permet aux organisations publiques de mieux innover? Il semble que la relation entre l'autonomie et l'innovation ne constitue pas une simple corrélation positive et linéaire. Les organismes autonomes semblent avoir l'avantage en ce qui a trait à l'innovation : ils produisent une bonne part des innovations, au nombre desquelles plusieurs sont suffisamment impressionnantes pour être récompensées par un prix, beaucoup plus que dans le cas des autres organisations. Cependant, les sociétés d'État, qui jouissent d'une plus grande autonomie, ne parviennent pas à profiter de cette situation pour créer de meilleures ou de plus nombreuses innovations. De plus, les organismes centraux pourraient bien constituer le second groupe le plus innovateur, malgré la très grande proximité des politiques de l'appareil d'État. Est-ce en raison du nombre de réformes affectant l'administration publique au cours des dernières décennies (que certains organismes centraux peuvent se vanter d'avoir pilotées) ou plutôt parce que les réformes sur lesquelles ils travaillent ont un effet plus vaste et retiennent mieux l'attention?

Selon Considine et Lewis (2007), le rôle des structures est controversé : est-ce le réseau ou la position constitutionnelle qui compte le plus? La centralisation a fait partie des éléments étudiés par Damanpour (1991) et nos résultats confirment ceux de Damanpour (1991, p. 567) quant aux organisations prestataires de services qui se trouvent en association positive entre la spécialisation, la différentiation fonctionnelle et l'innovation, dès lors que l'on prend en compte les organismes autonomes. Les sociétés d'État sont étonnantes. Il faudrait peut-être une théorie plus sophistiquée pour approcher le lien entre autonomie et innovation de manière à expliquer ce qui se produit dans leur cas. Selon Bernier et Hafsi (2007), les sociétés d'État auraient dû être plus innovatrices, mais la relation avec l'État n'est pas représentée seulement par l'éloignement quasi physique entre l'État et l'organisation. Dans leur étude d'une de ces relations, Hafsi et Konig (1988) ont suggéré la présence d'un cycle. Lorsqu'une organisation vient de voir le jour, elle est dominée par la logique et la vision de l'État. Elle reçoit également la plupart de ses ressources de l'État. Les principaux gestionnaires sont eux-mêmes d'anciens fonctionnaires, sensibles aux objectifs de l'État et ont parfois été des intervenants importants dans le développement de ces objectifs. Ils sont familiers avec la bureaucratie et sont respectés par la plupart des fonctionnaires avec lesquels ils sont en interaction. Les deux parties se comprennent et coopèrent sur la plupart des questions. C'est la phase de coopération, décrite également en tant que période au cours de laquelle la technologie de base d'une organisation se trouve encore dans ses années d'incubation.

Lorsque la technologie de base d'une organisation est en place, ses gestionnaires gardent les fonctionnaires à distance, de manière à les empêcher d'agir sur les normes rationnelles du noyau technologique de leur organisation (Thompson, 1967). Lorsque l'organisation grandit et que sa technologie de base progresse, la relation devient plus conflictuelle, les gestionnaires de l'organisation tentent de protéger leur technologie des interventions des hauts fonctionnaires. Avec le temps, comme le conflit prend de l'ampleur et coûte de plus en plus cher à toutes les parties, les hauts fonctionnaires tendent à s'en éloigner, octroyant ainsi plus d'autonomie à l'organisation. C'est un processus lent et progressif qui entraîne des confrontations. Celles-ci sont nombreuses, selon Hafsi et Kœnig (1988), mais d'ampleur limitée, et montrent une croissance sur le plan de l'intensité et de la fréquence. Les confrontations sont toutes liées aux efforts des gestionnaires pour protéger leur noyau technologique et son bon fonctionnement. Dans la troisième phase, les intenses confrontations sont de 
plus en plus coûteuses sur le plan politique et parfois institutionnel. Résultat, les fonctionnaires s'en éloignent et laissent l'organisation poursuivre une logique orientée vers le marché. Elle devient autonome de facto, en particulier lorsqu'il est possible de générer les ressources nécessaires aux activités régulières, ce que certaines sociétés d'État parviennent à accomplir.

La culture des entreprises pourrait également expliquer le nombre de candidatures soumises par les entreprises publiques. Comme c'est le cas du ministère des Affaires étrangères, les gestionnaires de ces entreprises ne se considèrent pas à priori comme des fonctionnaires.

Malgré toutes les précautions possibles dans l'interprétation de ces résultats, nous croyons que la relation qui a fait l'objet de nos tests est intéressante. Bien que l'innovation provienne de parties éloignées du cœur de l'appareil d'État, les innovateurs sont principalement des gestionnaires postés au milieu de l'échelle hiérarchique, comme le suggère Borins. Osborne et Brown (2005, p. 154) ont écrit que «la question de la capacité d'innover des organisations publiques n'a pas fait l'objet de recherches suffisantes ». Avec le présent article, nous espérons avoir aidé à suggérer une autre parcelle de l'explication. Le NMP proposait de remplacer les ministères par des organisations autonomes. La spécialisation des tâches est censée mener à des gains sur le plan de l'efficacité. Cela n'était peut-être pas une si bonne idée en général, mais pour ce qui est de l'innovation, les chantres du NMP avaient peut-être raison. Le mouvement vers l'autonomie des organisations a peut-être constitué un phénomène important. Ce processus était censé accroître l'efficacité, la gestion professionnelle, la proximité des services avec les citoyens et la capacité des ministres à se concentrer sur de plus importantes questions politiques et réduire les interventions politiques (Pollitt et autres, 2004, p. 3). Le mouvement vers l'autonomie des organisations a engendré de nouveaux problèmes de coordination (Bouckaert, Peters et Verhoest, 2010), mais semble avoir favorisé l'innovation.

\section{BIBLIOGRAPHIE}

Albury, D. (2005). «Fostering Innovation in Public Services», Public Money and Management, vol. 25 , no 1 , p. 51-56.

Arnaboldi, M., G. Azzone et T. Palermo (2010). « Managerial Innovations in Central Government: Not Wrong, but Hard to Explain », International Journal of Public Sector Management, vol. 23, $\mathrm{n}^{\mathrm{o}}$ 1, p. 78-93.

Bance, P. et L. Bernier (2011). Contemporary Crisis and Renewal of Pubic Action: Towards the Emergence of a New Form of Regulation?, Bruxelles, Peter Lang.

Bartunek, J. M. et autres (2007). "Front and Backstages of the Diminished Routinization of Innovations: What Innovation Research Makes Public and Organizational Research Finds Behind the Scenes », Strategic Entrepreneurship Journal, vol. 1, n 3-4, p. 295-314.

Bernier, L. et S. Angers (2010). "Le NMP ou le nouveau management public », dans S. Paquin, L. Bernier et G. Lachapelle (dir.), L'analyse des politiques publiques, Montréal, Presses de l’Université de Montréal, p. 229-254.

Bernier, L. et L. Farinas (2010). « Les entreprises publiques du gouvernement du Canada », dans R. Bernier (dir.), L'espace canadien: mythes et réalités, Québec, Presses de l'Université du Québec, p. 403-425. 
Bernier, L. et T. Hafsi (2007). "The Changing Nature of Public Entrepreneurship », Public Administation Review, vol. 67, no 3, p. 488-503.

Bernier, L., K. Brownsey et M. Howlett (dir.) (2005). Executive Styles in Canada, Toronto, University of Toronto Press.

Borins, S. (dir.) (2008). Innovations in Government, Washington D.C., The Brookings Institution Press.

Borins, S. (2001). The Challenge of Innovating in Government, Washington D.C., IBM Center for Business in Government.

Borins, S. (2000). «Public Service Awards Programs: An Exploratory Analysis », Canadian Public Administration, vol. 43, n 3, p. 321-342.

Borins, S. (1998). Innovating with Integrity: How Local Heroes Are Transforming American Government, Washington D.C., Georgetown University Press.

Borins, S. (1995). «Public Sector Innovation: The Implications of New Forms of Organization and Work », dans B. G. Peters et D. J. Savoie (dir.), Governance in a Changing Environment, Montréal, Mc-Gill-Queen's University Press, p. 260-287.

Borins, S. et R. M. Walker (2012). Many Are Called but Few Are Chosen: Modeling the Selection Process for the Innovations in American Government Awards, Ash Center for Democratic Governance and Innovation, Harvard Kennedy School.

Bouckaert, G., B. G. Peters et K. Verhoest (2010). The Coordination of Public Sector Organizations, London, Palgrave Macmillan.

Boyne, G. A. et autres (2005). «Explaining the Adoption of Innovation: An Empirical Analysis of Public Management Reform », Environment and Planning C: Government and Policy, vol. 23, no 3, p. 419-435.

Considine, M. et J. M. Lewis (2007). «Innovation and Innovators Inside Government: From Institutions to Networks », Governance: An International Journal of Policy, Administration, and Institutions, vol. 20, no 4, p. 581-607.

Crossan, M. M. et M. Apaydin (2010). «A Multi-Dimensional Framework of Organizational Innovation: A Systematic Review of the Literature », Journal of Management Studies, vol. 47, no 6, p. 1154-1191.

Currie, G. et autres (2008). «Entrepreneurial Leadership in the English Public Sector: Paradox or Possibility », Public Administration, vol. 86, n 4, p. 987-1008.

Damanpour, F. (1991). « Organizational Innovation: A Meta-Analysis of Effects of Determinants and Moderators », The Academy of Management Journal, vol. 34, nº 3, p. 555-590.

Damanpour, F. et J. D. Wischnevsky (2006). «Research on Innovation in Organizations: Distinguishing Innovation-Generating from Innovation-Adopting Organizations », Journal of Engineering and Technology Management, vol. 23, nº 4, p. 269-291.

Damanpour, F. et M. Schneider (2009). « Characteristics of Innovation and Innovation Adoption in Public Organizations: Assessing the Role of Managers », Journal of Public Administration Research and Theory, vol. 19, no 3, p. 495-522.

Denis, J-L et autres (2002). «Explaining Diffusion Patterns for Complex Health Care Innovations », Health Care Management Review, vol. 27, n 3, p. 60-73.

Dougherty, D. et D. D. Dunne (2011). "Organizing Ecologies of Complex Innovation», Organization Science, vol. 22, n 5, p. 1214-1223. 
Dunleavy, P. et autres (2006). "New Public Management is Dead: Long Live Digital-Era Governance », Journal of Public Administration Research and Theory, vol. 16, n 3, p. 467-494.

Dunn, C. (2010). The Handbook of Canadian Public Administration, Toronto, Oxford University Press.

Fernández, S. et L. R. Wise (2010). "An Exploration of Why Public Organizations "Ingest" Innovations », Public Administration, vol. 88, nº 4, p. 979-998.

Galimberti, J. (2003). Chronicling Public Sector Renewal in Canada: The IPAC Award for Innovative Management, United Nations, Economic and Social Council, Economic and Social Commission for Western Asia, Beirut, 1-3 juillet.

Glor, E. (2002). Is Innovation a Question of Will or Circumstance? An Exploration of the Innovation Processes Through the Lens of the Blakeney Government in Saskatchewan, 2e éd., Ottawa, The Innovation Journal.

Glor, E. (1998). "Public Sector Innovation in Canada», dans R. Hoffman et autres, Public Administration, Canadian Materials, 3e éd., Toronto, Cactus Press, p. 300-340.

Golden, O. (1990). "Innovation in Public Sector Human Services Programs: The Implication of Innovation by "Groping Along" », Journal of Policy Analysis and Management, vol. 9, n 2, p. 219-248.

Gow, J. I. (1994). Learning from Others: Administrative Innovations Among Canadian Governments, Toronto, Institut d'administration publique du Canada.

Gow, J. I. (1992). « Diffusion of Administrative Innovations in Canadian Public Administrations », Administration and Society, vol. 23, no 4, p. 430-454.

Hafsi, T. et C. Koenig (1988). "The State-SOE Relationship: Some Patterns », Journal of Management Studies, vol. 25, no 3, p. 235-249.

Hartley, J. (2005). "Innovation in Governance and Public Services: Past and Present », Public Money and Management, vol. 25, no 1, p. 27-34.

Hartley, J. et J. Downe (2007). "The Shining Lights? Public Service Awards as an Approach to Service Improvement », Public Administration, vol. 85, n 2, p. 329-353.

Hellström, T. (2004). «Innovation as Social Action », Organization, vol. 11, n 5, p. 631-649.

Janssen, O., E. van de Vliert et M. West (2004). « The Bright and Dark Sides of Individual and Group Innovation: A Special Issue Introduction », Journal of Organizational Behavior, vol. 25, $n^{\circ} 2$, p. 129-145.

Kernaghan, K. et D. Siegel (1999). Public Administration in Canada, 4e éd., Scarborough, Nelson.

Kernaghan, K., B. Marson et S. Borins (2000). The New Public Organization, Toronto, Institut d'administration publique du Canada.

Landau, J. (1993). «Organizational Change and Barriers to Innovation: A Case Study in the Italian Public Sector », Human Relations, vol. 46, no 12, p. 1411-1429.

Lekhi, R. (2007). Public Service Innovation: A Research Report for the Work Foundation's Knowledge Economy Programme, London, The Work Foundation.

Metcalfe, L. (1993). « Conviction Politics and Dynamic Conservatism: Mrs. Thatcher's Managerial Revolution », International Political Science Review, vol. 14, n 4, p. 351-371.

Miller, D. (1998). «Configurations Revisited», Strategic Management Journal, vol.17, n 7, p. 505-512. 
Mohamed, M. A. K. (2002). "Assessing Determinants of Departmental Innovation: An Explanatory Multi-Level Approach », Personnal Review, vol. 31, n 5, p. 620-641.

Moore, M. H. (2005). « Break-Through Innovations and Continuous Improvement: Two Different Models of Innovative Processes in the Public Sector », Public Money and Management, vol. 25, no 1 , p. 43-50.

Nohria, N. et R. Gulati (1996). « Is Slack Good or Bad for Innovation? », Academy of Management Journal, vol. 39, n 5, p. 1245-1264.

Osborne, S. P. et L. Brown (2011). «Innovation, Public Policy and Public Services Delivery in the UK: The Word that Would Be King? », Public Administration, vol. 89, n 4, p. 1335-1350.

Osborne, S. P. et K. Brown (2005). Managing Change and Innovation in Public Service Organization, London, Routledge.

Pollitt, C. et autres (2004). Agencies: How Governments Do Things Through Semi-Autonomous Organizations, London, Palgrave Macmillan.

Rangarajan, N. (2008). «Evidence of Different Types of Creativity in Government: A Multimethod Assessment », Public Performance and Management Review, vol. 32, nº 1, p. 132-163.

Richmond, D. et D. Siegel (dir.) (1994). Agencies, Boards and Commissions, Toronto, Institut d'administration publique du Canada.

Riccucci, N. M. (1995). Unsung Heroes: Federal Execucrats Making a Difference, Washington D.C., Goergetown University Press.

Roberts, A. (2010). "A Fragile State: Federal Public Administration in the 20 ${ }^{\text {th }}$ Century », dans C. Dunn, The Handbook of Canadian Public Administration, Toronto, Oxford University Press, p. 219-234.

Savoie, D. J. (1999). Governing from the Centre: The Concentration of Power in Canadian Politics, Toronto, Toronto University Press.

Skocpol, T. et K. Finegold (1982). «State Capacity and Economic Intervention in the Early New Deal », Political Science Quarterly, vol. 97, n² 2, p. 255-278.

Swan, J. et H. Scarbrough (2005). «The Politics of Networked Innovation », Human Relations, vol. 58, no 7, p. 913-43.

Tardi, G. (2010). «Departments and Other Institutions of Government», dans C. Dunn, The Handbook of Canadian Public Administration, Toronto, Oxford University Press, p. 25-51.

Thompson, J. D. (1967). Organization in Action, New York, Mc Graw Hill.

Walker, R. M. (2012). Internal and External Antecedents of Process Innovation: A Review and Extension, Mimeo, 12 juin.

Walker, R. M. (2007). «An Empirical Evaluation of Innovation Types and Organizational and Environmental Characteristics: Towards a Configuration Framework », Journal of Public Administration Research and Theory, vol. 18, no 4, p. 591-615.

Walker, R. M., F. Damanpour et C. A. Devece (2010). "Management Innovation and Organizational Performance: The Mediating Effect of Performance Management », Journal of Public Administration, Research and Theory, vol. 21, no 2, p. 367-386.

White, G. (2005). Cabinets and First Ministers, Vancouver, University of British Columbia Press.

Zussman, D. (2010). «Alternative Service Delivery in Canada », dans C. Dunn, The Handbook of Canadian Public Administration, Toronto, Oxford University Press, p. 250-267. 